\title{
Improvement of the interaction formulae for beam-columns in Eurocode 3
}

\author{
N. Boissonnade ${ }^{1}$, J.-P. Jaspart ${ }^{2}$, J.-P. Muzeau ${ }^{1 *}$, M. Villette $^{3}$ \\ ${ }^{1}$ LERMES / CUST, Blaise Pascal University, BP 206, 63174 AUBIÈRE Cedex, France \\ ${ }^{2}$ MSM, Institut du Génie Civil, University of Liège (Sart-Tilman), 4000 LIÈGE 1, Belgium \\ ${ }^{3}$ Baudin Châteauneuf S.A., 247 boulevard Thiers - BP 4131, 37041 TOURS Cedex, France
}

\begin{abstract}
This paper presents a new proposal for beam-column interaction formulae initially based on second-order in-plane elastic theory, as an alternative to those proposed in the Eurocode 3 pre-standard [1]. It has been derived according to the following requirements: theoretical background, clear physical meaning, consistency with the other related formulae of Eurocode 3 and accuracy. Besides that, the suggested formulae cover all required continuities: between the cross-section classes, from plasticity to elasticity as slenderness and axial force increase, and continuity between all the individual stability member checks and cross-section verifications. Further to the presentation of the formulae and their background, the good agreement of the proposal is shown through an extensive comparison with more than 15000 results of finite element numerical simulations.
\end{abstract}

Keywords: Instability - Buckling - Biaxial bending - Interaction - Beam-column Elastic-plastic - FEM numerical simulation 


\section{Introduction}

The stability of structural members subjected to combined axial compression forces and bending moments is a quite important problem for designers and it has been extensively studied during the last 50 years [2]; nevertheless, as a full satisfactory solution is still not yet available for daily practice, and because of the general tendency to increase the slenderness of steel members and frames, further research investigations have to be carried out with the aim to include safe and economic calculation rules in modern design codes. As far as Eurocode 3 is concerned, it is now widely recognised that the interaction formulae for beam-columns have to be significantly improved, in terms of accuracy and physical background. Present paper aims at describing a new proposal for beam-column interaction formulae, that could be an alternative to the Eurocode 3 ones. The proposed formulation has been derived in order to fulfil several objectives: economy and accuracy, generality, physical transparency and consistency with all the individual stability member checks (flexural instability in compression, lateral torsional buckling in bending...) and cross-section resistance verifications; it is based on a second-order in-plane elastic theory, and has been progressively extended to spatial and elastic-plastic behaviour. Its theoretical format is such that each constitutive coefficient is normally associated to a single physical effect; when this is not possible, results of FEM numerical simulations are used to calibrate locally some coefficients.

In this paper, for sake of simplicity and lack of space, attention will only be devoted to the design of members where lateral torsional buckling is not likely to occur, i.e. torsional deformations are prevented. But the out-of-plane behaviour of the beamcolumns subjected to biaxial bending and axial compression will be considered. This case is not a common one in practice, except for tubular construction, but it represents an important step for the development of the presented formulae. Complete formulae 
including lateral torsional buckling effects are available and have been also validated through comparisons with FEM simulations; the interested reader is kindly requested to refer to references [3] and [4] for more details.

Finally, notations used in the paper are those suggested in Eurocode 3.

\section{Basis of the formulation: second-order in-plane elasticity}

\subsection{Simply supported member under axial compression}

In Fig 1, a simply supported member under pure compression with an initial transverse deflection is illustrated. The initial deflection is supposed to be sinusoidal, and may therefore be expressed as:

$$
v_{0}(x)=e_{0, d} \sin \frac{\pi x}{L}
$$

For an applied axial compression $N_{S d}$, the additional deflection $v(x)$ can be evaluated by solving the following classical buckling equilibrium equation:

$$
v(x)=\frac{N_{S d}}{N_{c r}-N_{S d}} e_{0, d} \sin \frac{\pi x}{L},
$$

where $N_{c r}$ is the Euler elastic critical load. The total deflection at mid-span of the beam then amounts $e_{0, d}+v(L / 2)$ and the resistance criterion of the member crosssection at mid span including second-order effects is then expressed:

$$
\frac{N_{S d}}{N_{p l . R d}}+\frac{1}{1-N_{S d} / N_{c r}} \frac{N_{S d} e_{0, d}}{M_{e l . R d}} \leq 1
$$

In Eq. (3), $M_{e l . R d}$ and $N_{p l . R d}$ respectively represent the elastic bending resistance and the plastic resistance in compression of the cross-section. Keeping in mind that, at 
failure, the maximum applied axial force reaches the actual buckling resistance $N_{b . R d}=\chi N_{p l . R d}$ of the column, the value of $e_{0, d}$ may be derived:

$$
e_{0, d}=\frac{(1-\chi)\left(1-\chi N_{p l . R d} / N_{c r}\right)}{\chi} \frac{M_{e l . R d}}{N_{p l . R d}} .
$$

\subsection{Beam-column: in-plane behaviour}

Eq. (3) can easily be extended to the case of beam-columns by adding a term covering the effect of the distribution of first order bending moments in the member:

$$
\frac{N_{S d}}{N_{p l . R d}}+\frac{1}{1-N_{S d} / N_{c r}} \frac{N_{S d} e_{0, d}}{M_{e l . R d}}+\frac{1}{1-N_{S d} / N_{c r}} \frac{C_{m} M_{S d}}{M_{e l . R d}} \leq 1,
$$

In this expression, two new concepts are introduced: the equivalent moment factor $C_{m}$ (see paragraph 2.2.2), and the amplification factor $1 /\left(1-N_{S d} / N_{c r}\right)$ (see paragraph 2.2.1). By considering the value of $e_{0, d}$ derived from (4), Eq. (5) may be written under several formats:

- $\chi *$ format [5]:

$$
\frac{N_{S d}}{\chi^{*} N_{p l . R d}}+\frac{C_{m} M_{S d}}{\left(1-N_{S d} / N_{c r}\right) M_{e l . R d}} \leq 1
$$

where:

$$
\frac{1}{\chi^{*}}=1+\frac{(1 / \chi-1)\left(1-\chi \bar{\lambda}^{2}\right)}{\left(1-N_{S d} / N_{c r}\right)} .
$$

- With a $\Delta n$ term, as in DIN 18800 [6]:

$$
\frac{N_{S d}}{\chi N_{p l . R d}}+\frac{C_{m} M_{S d}}{M_{e l . R d}} \leq 1-\Delta n
$$


where:

$$
\Delta n=\frac{N_{S d}}{N_{p l . R d}}\left(1-\frac{N_{S d}}{\chi N_{p l . R d}}\right) \chi^{2} \bar{\lambda}^{2} .
$$

- $\mu$ format:

$$
\frac{N_{S d}}{\chi N_{p l . R d}}+\mu \frac{C_{m} M_{S d}}{\left(1-N_{S d} / N_{c r}\right) M_{e l . R d}} \leq 1,
$$

with:

$$
\mu=\frac{1-N_{S d} / N_{c r}}{1-\chi N_{S d} / N_{c r}}
$$

The last format is the one adopted in the proposal as the first term of (10) simply corresponds to the Eurocode 3 stability check for a member in pure compression.

\subsubsection{Amplification factor}

The distribution of first order bending moments along the member is affected by the application of the axial compression force because of well-known second order effects. For a member subjected to a sinusoidal distribution of first order bending moments, the “amplified" moments resulting from the application of the axial force $N_{S d}$ are obtained by multiplying the first order bending moments by the following amplification factor already used in section 2.1: $\quad \frac{1}{1-N_{S d} / N_{c r}}$,

For sake of simplicity, the same amplification factor has been applied in section 2.2 to the $M_{S d}$ moment distribution, what is not strictly true from a scientific point of view. For instance, for a column subjected to end moments (linear distribution of first order bending moments along the column, the exact value of the amplification factor is [7]:

$$
\frac{1}{\cos \frac{\pi}{2} \sqrt{N_{S d} / N_{c r}}}
$$

This second expression gives quite similar results than Eq. (12) when the axial force is 
far from a critical state (i.e. $N_{S d} / N_{c r}$ is small), as shown in Fig. 2. But the difference between these two theoretical expressions becomes significant when $N_{S d}$ gets closer to the critical value, and it is then necessary to account for this influence in Eq. (5), (6), (8) and (10). In the next paragraph, it will be shown that this correction may be taken into consideration through the adoption of appropriate values for the equivalent moment factor $C_{m}$.

\subsection{2. $C_{m}$ coefficient}

The concept of "equivalent moment" is a quite usual one as far as beam-columns are concerned. It simply allows replacing the actual distribution of first order bending moments along the member by a sinusoidal equivalent one.

The maximum first order moment in the equivalent moment distribution is defined as $C_{m} M_{S d}$; in this expression, $M_{S d}$ is the value of the maximum moment in the actual first order distribution of bending moments, and $C_{m}$ is called "equivalent moment factor”. The equivalence is such that the maximum amplified moment resulting from the application of the axial compression force in the actual member is equal to the maximum amplified moment in a similar column subjected to the sinusoidal equivalent moment distribution. This is illustrated in Fig. 4.

This concept brings significant simplification as the maximum amplified equivalent moment is located at mid-span.

From theoretical considerations [6], the exact expressions of $C_{m}$ to apply to a member subjected to a linear first order bending moment distribution (i.e. $M_{S d}$ at one end of the member and $\psi M_{S d}$ at the other end, $-1 \leq \psi \leq 1$ ), are: 


$$
C_{m}=\left(1-\frac{N_{S d}}{N_{c r}}\right) \frac{\sqrt{1-2 \psi \cos \left(\pi \sqrt{N_{S d} / N_{c r}}\right)+\psi^{2}}}{\sin \left(\pi \sqrt{N_{S d} / N_{c r}}\right)}
$$

when $N_{S d} \geq N_{\text {lim }}$, and:

$$
C_{m}=1-\left(\frac{\arccos \psi}{\pi}\right)^{2}
$$

when $N_{S d}<N_{\lim }$, where $N_{\lim }=N_{c r}\left(\frac{\arccos \psi}{\pi}\right)^{2}$ corresponds to the value under which the collapse is reached by lack of resistance at the end of the member, and not by instability phenomena.

Villette [5], [8], extensively studied the problem, and derived a simple approximate expression, which allows, in Eq. (10), to account for the theoretical error mentioned in $\S 2.2 .1$ :

$$
C_{m}=0,79+0,21 \psi+0,36(\psi-0,33) \frac{N_{S d}}{N_{c r}}
$$

It is worthwhile to state that the combined use of Eq. (10) and (16) does not prevent the designer from checking the resistance of the member end cross-sections.

Eq. (16) has been integrated in the proposal. Fig. 3 outlines the fundamental differences between the theoretical value of $C_{m}$ and some other well-known definitions, i.e. the Austin one:

$$
C_{m}=0,6+0,4 \psi \geq 0,4
$$

and the Massonnet one:

$$
C_{m}=\sqrt{0,3\left(1+\psi^{2}\right)+0,4 \psi} \text {. }
$$

Besides the fact that the theoretical values of $C_{m}$ depend on $N_{S d} / N_{C r}$ values, a major 
difference between Eqs. (16) and (17) or (18) lies in their opposite curvatures, as clearly seen in Fig. 3. This difference may probably be explained, at least for expression (18), by the fact that the latter has been initially developed to cover the influence of the actual bending moment distribution on the value of the elastic critical moment $M_{C r}$ for lateral torsional buckling (Eurocode $3 C_{1}$ coefficient) and then extended to beamcolumns.

For beam-columns subjected to transverse loading or end moments plus transverse loading, it is recommended to adopt the following value for the equivalent moment factor $C_{m}[7]$ :

$$
C_{m}=1+\left(\frac{\pi^{2} E I v_{0}}{M_{0} L^{2}}-1\right) \frac{N_{S d}}{N_{c r}},
$$

where the index " 0 " means "first order". When the member is laterally restrained by means of one or more intermediate supports, $C_{m}$ must be lower bounded to $1-N_{S d} / N_{c r}$, because the amplified moments are unknown along the beam.

The validity of this new definition of the equivalent moment factor is shown in $\S 5$.

In the next section, the formulae (10) are extended to column subjected to biaxial bending moments and modified to integrate plasticity effects.

\section{Extension to spatial behaviour and plasticity}

\subsection{Spatial behaviour}

The in-plane format of Eq. (10) may be easily extended to the more usual case of beamcolumn subjected to biaxial bending moments and spatial instability. For each instability plane i (y or z according to Eurocode 3), the following equation may be 
applied:

$\frac{N_{S d}}{\chi_{i} N_{p l . R d}}+\mu_{i}\left[\frac{C_{m . y} M_{y . S d}}{\left(1-N_{S d} / N_{\text {cr.y }}\right) M_{\text {el.y.Rd }}}\right]+\mu_{i}\left[\frac{C_{m . z} M_{z . S d}}{\left(1-N_{S d} / N_{\text {cr.z }}\right) M_{\text {el.z.Rd }}}\right] \leq 1(20)$

\subsection{Elastic-plastic behaviour}

Eq. (20) may be generalised to the case of Class 1 and 2 cross-sections (sections which may develop their full plastic moment resistance in bending without premature local plate buckling of the constitutive walls in compression, according to Eurocode 3), by simply replacing the elastic bending moment resistance $M_{e l . R d}$ by an elastic-plastic one noted $k M_{p l . R d} ; k$ is a plasticity coefficient expressed as follows:

$$
k=1+(w-1)\left[2-\frac{1,6}{w} C_{m}^{2}\left(\bar{\lambda}+\bar{\lambda}^{2}\right)\right] \frac{N_{S d}}{N_{p l . R d}} \geq \frac{W_{e l}}{W_{p l}}
$$

with: $\quad w=\frac{W_{p l}}{W_{e l}} \leq 1,5$.

$W_{p l}$ and $W_{e l}$ designate respectively the plastic and elastic modulus of the column crosssection in bending. $\bar{\lambda}$ represents the reduced column slenderness for flexural instability. Because of instability effects, it appears that the beam cannot develop its full plastic capacity, that must then be tempered by this $k$ coefficient. The definition of $k$ must also depend on $\bar{\lambda}$, to allow the behaviour of the beam being plastic for small slenderness, and to become elastic as $\bar{\lambda}$ and axial compression increase, as it is in reality. In Eq. (21), the factor $(w-1)$ represents the maximum available bending potential between pure elasticity and pure plasticity, and must be multiplied not only by a function of $\bar{\lambda}$, as explained before, but also by a function of $C_{m}$, because the member cannot develop the same elastic-plastic effects whatever the transverse loading is. This 
calibrated coefficient then clearly permits a smooth physical transition between plasticity and elasticity; it will be seen in $\S 4$ that Eq. (21) is consistent with a crosssection check, i.e. when the slenderness tends to 0 .

The bending moment of the column cross-sections being always greater than the elastic moment resistance, the $k$ coefficient must be bounded by $W_{e l} / W_{p l}$ for Class 1 and 2 cross-sections, and by $W_{e l} / W_{3}$ for Class 3 cross-sections (sections which, according to Eurocode 3, are only able to develop a bending moment resistance equal to the full elastic one), $W_{3}$ being an intermediate plasticity modulus allowing a continuous transition between Class 2 and 3 cross-sections (cf. § 4.3).

\subsection{Biaxial bending}

In the particular case of biaxial bending, Eq. (20) writes:

$$
\frac{C_{m . y} M_{y . S d}}{M_{p l . y . R d}}+\frac{C_{m . z} M_{z . S d}}{M_{p l . z . R d}} \leq 1,
$$

Eq. (23) accounts also for plasticity effects, as detailed in the previous paragraph; this is a linear interaction criterion, while, from a physical point of view, it should not. For instance, the Eurocode 3 formula (Eq. 24) is not linear:

$$
\left(\frac{M_{y . S d}}{M_{\text {pl.y.Rd }}}\right)^{\alpha}+\left(\frac{M_{z . S d}}{M_{\text {pl.z.Rd }}}\right)^{\beta} \leq 1 .
$$

Then, the formulation must be modified in order to take this effect into account. This is achieved in decoupling Eq. (20) verification as follows:

$$
\begin{gathered}
\frac{N_{S d}}{\chi_{y} N_{p l . R d}}+\mu_{y}\left[\frac{C_{m . y} M_{y . S d}}{\left(1-N_{S d} / N_{c r . y}\right) k_{y y} M_{p l . y . R d}}\right], \\
+\alpha^{*} \mu_{y}\left[\frac{C_{m . z} M_{z . S d}}{\left(1-N_{S d} / N_{c r . z}\right) k_{y z} M_{p l . z . R d}}\right] \leq 1
\end{gathered}
$$




$$
\begin{gathered}
\frac{N_{S d}}{\chi_{z} N_{p l . R d}}+\beta^{*} \mu_{z}\left[\frac{C_{m . y} M_{y . S d}}{\left(1-N_{S d} / N_{c r . y}\right) k_{z y} M_{p l . y . R d}}\right], \\
\quad+\mu_{z}\left[\frac{C_{m . z} M_{z . S d}}{\left(1-N_{S d} / N_{c r . z}\right) k_{z z} M_{p l . z . R d}}\right] \leq 1
\end{gathered}
$$

where $\alpha^{*}$ and $\beta^{*}$ are coefficients accounting for $M_{y}-M_{z}$ plastic interaction. Eq. (25) is then a strong axis check, while Eq. (26) is a weak axis one; consequently, in the particular case of pure biaxial bending, Eqs. (25) and (26) reduce as:

$$
\begin{aligned}
& \frac{C_{m . y} M_{y . S d}}{M_{p l . y . R d}}+\alpha * \frac{C_{m . z} M_{z . S d}}{M_{p l . z . R d}} \leq 1, \\
& \beta^{*} \frac{C_{m . y} M_{y . S d}}{M_{p l . y . R d}}+\frac{C_{m . z} M_{z . S d}}{M_{p l . z . R d}} \leq 1,
\end{aligned}
$$

This new formulae allows increasing the accuracy of the proposal in the case of biaxial bending, as it is shown in Fig. 5.

$\alpha^{*}$ and $\beta^{*}$ are respectively chosen as $0,6 \sqrt{w_{z} / w_{y}}$ and $0,6 \sqrt{w_{y} / w_{z}}$, to allow dealing with any kind of cross-sections.

Because of the spatial behaviour, the $k_{i i}$ coefficients must write:

$$
k_{i i}=1+\left(w_{i}-1\right)\left[2-\frac{1,6}{w_{i}} C_{m . i}{ }^{2}\left(\bar{\lambda}_{\max }+\bar{\lambda}_{\max }^{2}\right)\right] \frac{N_{S d}}{N_{p l . R d}},
$$

where $\bar{\lambda}_{\text {max }}$ is the maximum value of $\bar{\lambda}_{y}$ and $\bar{\lambda}_{z}$, and $k_{i i}$ bounded as in (21).

For the same reason as explained in $\S 3.2$, it becomes necessary to introduce two other $k_{i j}$ coefficients, calculated from calibration: 


$$
\begin{gathered}
k_{i j}=1+\left(w_{j}-1\right)\left[2-14 \frac{C_{m . j}{ }^{2} \bar{\lambda}_{\text {max }}^{2}}{w_{j}^{5}}\right] \frac{N_{S d}}{N_{p l . R d}}, \\
k_{i j} \geq 0,6 \sqrt{\frac{w_{j}}{w_{i}}} \frac{W_{e l}}{W_{p l}} \text { or } 0,6 \sqrt{\frac{w_{j}}{w_{i}}} \frac{W_{e l}}{W_{3}} .
\end{gathered}
$$

Eqs. (25) and (26) are the general formulae of the proposal. They allow to deal with biaxial and elastic-plastic behaviour, on the basis of in-plane second-order elasticity. They can also be adapted easily to lateral torsional buckling, but this case will not be presented in this paper (cf. [3], [4]). Because of their theoretical background, they present a strong physical meaning, preventing from mistakes in their use. Next paragraph aims at showing that this general shape reduces to well-known formulae when the applied loading is simple. It is details also how elastic-plastic effects can be integrated in a continuous way, as it is physically, but not in Eurocode 3 at that time.

\section{Continuity aspects}

One of the most important requirements for the development of such a formula lies in its ability to present as much continuities as possible. This paragraph is then devoted to point out continuity aspects, which can be divided into 3 types: continuity between plane and spatial behaviour, continuity between stability and cross-section resistance checks, and smooth resistance transitions in the elastic-plastic range, as a consequence of instability effects.

\subsection{Simple loading cases}

The general format of Eqs. (25) and (26) can reduce to more simple expressions, when one or more external forces vanish. The case where only two kinds of loading are effective on the beam is deduced from the general formulae by simply cancelling the 
appropriate term, like for example the case of axial compression with strong axis bending moment:

$$
\begin{gathered}
\frac{N_{S d}}{\chi_{y} N_{p l . R d}}+\frac{C_{m . y} M_{y . S d}}{\left(1-\chi_{y} N_{S d} / N_{c r . y}\right) k_{y y} M_{p l . y . R d}} \leq 1, \\
\frac{N_{S d}}{\chi_{z} N_{p l . R d}}+\frac{\beta^{*} \mu_{z} C_{m . y} M_{y . S d}}{\left(1-N_{S d} / N_{\text {cr.y }}\right) k_{z y} M_{p l . y . R d}} \leq 1 .
\end{gathered}
$$

In this particular case, the two checks of Eqs. (32) and (33) are still required, because the collapse of the beam can be either about its strong or its weak axis plane. It should be noticed that Eq. (33) clearly underlines the influence of strong axis bending about the weak axis buckling, which concept is not fully recognised in well-known standards.

Obviously, the case where the beam is loaded by in-plane forces only is covered, in considering only one equation, like Eq. (32) for example. If the beam is only submitted to axial compression for instance, the formulation simplifies into the Eurocode 3 buckling check:

$$
\frac{N_{S d}}{\min \left(\chi_{y}, \chi_{z}\right) N_{p l . R d}} \leq 1
$$

Of course, other cases like mono- or biaxial bending are covered by the formulation in the same way (cf. § 3.3). The proposal deals also with all cases of buckling stability problems, whatever the type of loading, i.e. axial compression and biaxial bending. It has been shown that it presents all types of continuities for this particular point, and next paragraph focuses on the continuities between stability and cross-section resistance checks.

\subsection{Stability to resistance continuity}

Another important aspect that the formulation should cover is linked with the influence 
of the length of the beam. Indeed, the stability verifications must reduce to crosssection resistance checks, as the buckling effects are no longer influencing the behaviour of the beam, i.e. when the slenderness tends to 0 . If pure compression, pure bending and biaxial bending cases are covered, the case of axial force with bending need to be further detailed. More, when the length of the beam tends to 0 , the verification becomes respectively a plastic or an elastic check, for Class 1 and 2, or Class 3 cross-sections. It has been shown in $\S 3.1$ that for pure elastic behaviour, Eq. (20) should be used. In this case, it reduces to:

$$
\frac{N_{S d}}{N_{\text {pl.Rd }}}+\frac{C_{m . y} M_{y . S d}}{M_{\text {el.y.Rd }}}+\frac{C_{m . z} M_{z . S d}}{M_{\text {el.z.Rd }}} \leq 1,
$$

This is the same elastic resistance check as in Eurocode 3 when first order moments reach their maximum value in span $\left(C_{m}=1\right)$. In other cases, the cross-section check becomes determinant before $\bar{\lambda}=0$, and so when $\bar{\lambda}=0$. But for Class 1 and 2 crosssections, plastic verifications can be made; and when the slenderness tends to 0 , the $k_{i i}$ coefficient expresses:

$$
k_{i i}=1+2\left(w_{i}-1\right) \frac{N_{S d}}{N_{p l . R d}} \geq \frac{W_{e l}}{W_{p l}},
$$

Eq. (36) shape allows to develop an intermediate bending resistance between $M_{p l . R d}$ and $M_{e l . R d}$, as drawn in Fig. 7 and 8. The value 2 in Eq. (36) is the highest integer one that can take the benefits of plasticity without being insecure when the axial force is high. 


\subsection{Particular Class 3 cross-section cases}

As already mentioned before, the proposal was developed so that the transitions between elasticity and plasticity are smooth and continuous, as it is actually. It has been shown in $\S 3.2$ that this is effective for a member, but this is not the case between crosssections classes. As shown in Fig. 6, according to Eurocode 3, a step of bending resistance exists between Class 2 and 3 cross-sections. So, the formulation proposes to adopt a new “elastic-plastic modulus $W_{3}$ ” allowing a smooth transition along Class 3 field. As this coefficient should depend on the $b / t$ ratios of the cross-sections walls, it is proposed to follow the Australian AS4100-1990 recommendations, that may be expressed as follows:

$$
W_{3}=W_{e l}+\left(W_{p l}-W_{e l}\right) \min \left[\frac{(b / t)_{3 . i}-(b / t)_{i}}{(b / t)_{3 . i}-(b / t)_{2 . i}}\right] .
$$

where $(b / t)_{2}$ is the boundary $b / t$ ratio between Class 2 and Class 3 for a particular cross-section wall, $(b / t)_{3}$ the one between Class 3 and Class 4 and index $i$ indicates each of the cross-section walls in full or partial compression. As a consequence of Eq. (37), the bending resistance of Class 3 cross-sections becomes a smooth linear transition between the plastic and the elastic behaviour (cf. Fig. 6).

\section{Results - Accuracy}

The formulae have been tested from more than 200 test results [5], and showed precise and safe results; but because of the large number of parameters involved in the complex behaviour of beam-columns, these tests cannot be sufficient to prove the accuracy of the proposal in all cases. Therefore, a comparison between the proposed formulae and numerical simulations has been performed (from about 15000 simulations). First, the 
numerical hypotheses and models are described here, and then some particular results are presented.

\subsection{Numerical models}

This paragraph gives a general overview of the calculations and a description of the assumptions made in the numerical model. Two software were used for the study: most of the results were carried out from ABAQUS [9], and some of them from FINELG [10] in the particular case of a IPE 200 shape. This allowed comparing both results, and these have been found in excellent agreement. Then, a parametric study was led, with:

1: 4 different profiles: IPE 200, IPE 500, HEB 300 and RHS $200 \times 100 \times 10$,

2: 4 values of the relative slenderness: $0.5,1.0,1.5$ and 3.0,

3: 5 types of primary bending moments diagrams: linear $(\psi=1)$, triangular ( $\psi=0$ ), bi-triangular $(\psi=-1)$, concentrated load at mid span and uniformly distributed load,

4: 3 types of loading: in-plane $y-y$, in-plane $z-z$ and biaxial bending combined with axial compression.

So, wide ranges of cases were studied, to cover as well as possible most of real practical cases.

Beam elements have been used: 100 using ABAQUS with 51 integration points over the cross-section, and 10 using FINELG with 25 integration points over the cross-section and 4 along each element.

The beams were considered simply supported, and initial sinusoidal deflections with maximum amplitude of $L / 1000$ were introduced in both principal planes. Regarding the material, an elastic - perfectly plastic constitutive law (i.e. without strain-hardening) 
has been used, with Fig. 10 characteristics. Additional imperfections such as residual stresses were also accounted, as described on Fig. 9.

The performed calculations followed a geometrical and material non-linear calculation model, which coupled with the hypotheses described above, allow the real behaviour of the member to be simulated as precisely as possible. For the particular case of biaxial loading, the degrees of freedom relative to torsional deformations have been restrained, in order not to have any interaction with lateral torsional buckling effects.

\subsection{In-plane behaviour}

Only results about in-plane behaviour are presented here, and they are restricted to weak axis bending. Considering that for classical I-section shapes, $M_{\text {pl.z.Rd }}$ is about $50 \%$ higher than $M_{e l . z . R d}$, while $M_{\text {pl.y.Rd }}$ is only about $15 \%$ higher than $M_{\text {el.y.Rd }}$, plasticity effects are much more important for in-plane weak axis bending (cf. Fig. 7 and 8). Nevertheless, the conclusions for strong axis in-plane bending are similar.

Tables 1 and 2 present the values for $R_{\text {simul }}$, the ratio between the loading giving failure according to the FEM simulations to the same proportional loading giving failure according to the proposal (cf. Eq. 38).

$$
R_{\text {simul }}=\frac{\left(N_{S d}, M_{y . S d}, M_{z . S d}\right)_{\text {failure, FEM }}}{\left(N_{S d}, M_{y . S d}, M_{z . S d}\right)_{\text {failure, proposal }}} .
$$

For instance, $R_{\text {simul }}=1.03$ when the exact failure loading according to the FEM simulations is $3 \%$ higher than the proportional one according to the proposal. Consequently, $R_{\text {simul }}$ shows the safety and the accuracy of the proposal. When $R_{\text {simul }} \geq 1$, that means that the proposal is safe. In the tables, the classical statistical values are presented: $m$ is the mean value, and $s$ is the standard deviation. 
As explained in $\S 2.2 .2$, the use of the $C_{m}$ coefficient concept in the formulation implies an additive cross-section check. The one considered in the numerical calculations [11] led sometimes to slightly unsafe values, that cannot be objected to the proposal. In addition, some of the numerical results were sometimes unsafe in the case of pure buckling, but never more than 3\%. Then, because the proposal intends only to deal with stability and $N-M$ interaction, these values do not characterise really the accuracy of the proposal. Then, some other results $R_{\text {simul }} \leq 0.97$ were also included in the tables, because they really represent unsafe values due to the proposal. Tables 1 and 2 show that the results are satisfactory: mean values are close to 1 , and the standard deviation is relatively small. The maximum value from about 979 results does not exceed 1.25 , without real unsafe value (the minimum is 0.967 ; in other words, less than $4 \%$ are on the unsafe side).

\subsection{Biaxial bending}

Besides the fact that the proposal also accounts for lateral torsional buckling [12], only results where torsional deformations are prevented are presented, i.e. the collapse has been reached by buckling or by end-sections excess of plasticity. Results including lateral torsional buckling will be presented later.

Here, the beam is subjected to biaxial bending with axial compression: having spatial components, the cross-section displacements are much more complex. As a consequence, more results are presented: cases where at failure $M_{z . S d} / M_{p l . z . R d}=0$, 0.2, 0.4, 0.6 and 0.8 are reported in Tables 3 and 4 .

The results are in a good agreement, accounting that because of biaxial effects, the behaviour of the member is relatively complex. Mean values are satisfactory as the maximum values stay under $25 \%$ safety. In order to emphasise the accuracy of the 
proposal for biaxial bending cases, Fig. 11 shows an example of interaction diagram.

\section{Conclusion}

The formulae for beam-columns detailed in this paper are based on second order inplane elasticity. This theoretical format allows the proposal to be physically understandable and general. It does not cover only plane behaviour but also spatial behaviour and loading, elastic-plastic effects and accounts for instability phenomenon. It was derived in order to present a maximum of continuities: between cross-section classes, from stability to resistance checks, between pure elastic and pure plastic behaviour, and with the other formulae of Eurocode 3.

In addition, several concepts such as amplification effects or equivalent moment concept are discussed, and new expressions are proposed.

A parametric study show that the proposal is safe and efficient, and that it is much more accurate than the one included in Eurocode 3.

Extensions to cases where lateral torsional buckling is possible are already available [3], [4], [12], but still need to be improved. This will be the next development. 


\section{References}

[1] Eurocode 3: Design of steel structures. Part 1-1, General rules and rules for buildings, ENV 1993-1-1, 1993.

[2] Massonnet C. 40 years of research on beam-columns in steel. Solid Mechanics Archives, 1976, 1(1).

[3] Villette M., Boissonnade N., Muzeau J.P. and Jaspart J.P. Development of a comprehensive formula for the design of beam-columns, Internal report, BaudinChâteauneuf, LERMES-CUST, University of Liege, 2000.

[4] Maquoi R., Boissonnade N., Muzeau J.P., Jaspart J.P. and Villette M. The interaction formulae for beam-columns: a new step of a yet long story. Proceedings of the 2001 SSRC Annual Technical Session \& Meeting, pp. 63-88, 2001.

[5] Bureau A., Galéa Y., Jaspart J.P., Maquoi R., Muzeau J.P. and Villette M. Proposal for a revision of Eurocode 3, TC8-ECCS meeting, Timisoara, 1999.

[6] DIN 18800, Teil 1, Stahlbauten, Bemessung und Konstruktion, Beuth Verlag GMBH, Berlin, 1988.

[7] Maquoi R., Rondal J. Sur la force portante des poutres colonnes, Annales des travaux publics de Belgique, 1982.

[8] Villette M. Considérations sur le flambement, proposition de révision de l’Eurocode 3, Construction Métallique, 1997, 3, 15-38.

[9] Ofner R. Traglasten Von Stäben aus Stahl bei Druck und Biegung, PhD Thesis, Institut für Stahlbau, Holzbau und Fläschentragwerke, Technische Universität 
Graz, Fakultät für Bauingenieurwesen, 1997.

[10] Boissonnade N. Contribution au développement de nouvelles formules pour éléments comprimés et fléchis au sens de l’Eurocode 3, Diploma work, MSM Liège, CUST, Blaise Pascal University (1999).

[11] Lescouarc’h Y. Capacité de résistance d'une section soumise à divers types de sollicitations, Construction Métallique, 1997, 2, 3-17.

[12] TC8-ECCS ad hoc working group on beam-columns, Background information on the "Level 2" beam-column design formulae and evaluation of its accuracy, TC8ECCS meeting, London, 2000. 


\section{Figure Captions}

Figure 1 Axially loaded member

Figure 2 Amplification factor

Figure 3 Comparison of different $C_{m}$ factors

Figure 4 Equivalent moment coefficient concept

Figure 5 Biaxial bending interaction for a classical IPE shape

Figure 6 Continuity between cross-section classes

Figure $7 \quad N-M_{y}$ cross-section interaction (HEB shape)

Figure $8 \quad N-M_{z}$ cross-section interaction (HEB shape)

Figure 9 Residual stress diagrams used in the simulations

Figure 10 Elastic-perfectly plastic constitutive law

Figure 11 Example of interaction diagram (IPE 500)

Abbreviated title of the paper:

"New interaction formulae for beam-columns in Eurocode 3" 
Table 1

Results for $Z-Z$ in-plane behaviour

IPE 200 FINELG

\begin{tabular}{lllllll}
\hline$\overline{\lambda_{z}}$ & 0,5 & 1,0 & 1,5 & 0,5 & 1,0 & 1,5 \\
\hline$m$ & 1,0374 & 1,0162 & 1,0254 & 1,0686 & 1,0378 & 1,0439 \\
\hline$s$ & 0,0368 & 0,0305 & 0,0307 & 0,0537 & 0,0395 & 0,0373 \\
\hline $\max$ & 1,1319 & 1,0747 & 1,1002 & 1,1760 & 1,1145 & 1,1082 \\
\hline min & 0,9696 & 0,9666 & 0,9776 & 0,9817 & 0,9695 & 0,9688 \\
\hline$\Sigma$ tests & 32 & 32 & 34 & 101 & 105 & 110 \\
\hline$\Sigma$ tests $<1$ & 1 & 9 & 7 & 5 & 22 & 13 \\
\hline Etests $<0,97$ & 1 & 2 & 0 & 0 & 1 & 2 \\
\hline
\end{tabular}

\section{Table 2}

Results for $Z-Z$ in-plane behaviour

\begin{tabular}{|c|c|c|c|c|c|c|c|c|c|}
\hline & \multicolumn{3}{|c|}{ IPE 500} & \multicolumn{3}{|c|}{ HEB 300} & \multicolumn{3}{|c|}{ RHS 200} \\
\hline$\overline{\lambda_{z}}$ & 0,5 & 1,0 & 1,5 & 0,5 & 1,0 & 1,5 & 0,5 & 1,0 & 1,5 \\
\hline$m$ & 1,0674 & 1,0356 & 1,0333 & 1,0248 & 1,0234 & 1,0311 & 0,9931 & 1,0203 & 1,0307 \\
\hline$S$ & 0,0558 & 0,0382 & 0,0375 & 0,0321 & 0,0330 & 0,0317 & 0,0126 & 0,0251 & 0,0302 \\
\hline $\max$ & 1,1902 & 1,1015 & 1,0977 & 1,0988 & 1,0899 & 1,0821 & 1,0317 & 1,0730 & 1,0918 \\
\hline $\min$ & 0,9816 & 0,9682 & 0,9689 & 0,9802 & 0,9687 & 0,9711 & 0,9748 & 0,9736 & 0,9784 \\
\hline Etests & 57 & 59 & 60 & 62 & 62 & 59 & 69 & 68 & 69 \\
\hline$\Sigma$ tests $<1$ & 5 & 12 & 14 & 15 & 18 & 12 & 46 & 12 & 10 \\
\hline$\Sigma$ tests $<0,97$ & 0 & 2 & 2 & 0 & 1 & 0 & 0 & 0 & 0 \\
\hline
\end{tabular}

Table 3

Results for spatial behaviour

\begin{tabular}{|c|c|c|c|c|c|c|c|}
\hline & \multicolumn{3}{|c|}{ IPE 200 FINELG } & \multicolumn{4}{|c|}{ IPE 200} \\
\hline$\overline{\lambda_{z}}$ & 0,5 & 1,0 & 1,5 & 0,5 & 1,0 & 1,5 & 3,0 \\
\hline$m$ & 1,0643 & 1,0562 & 1,0471 & 1,0926 & 1,0842 & 1,0737 & 1,0320 \\
\hline$s$ & 0,0443 & 0,0402 & 0,0339 & 0,0691 & 0,0541 & 0,0454 & 0,0345 \\
\hline $\max$ & 1,1611 & 1,1394 & 1,1236 & 1,2371 & 1,1957 & 1,1665 & 1,1182 \\
\hline $\min$ & 1,0000 & 0,9658 & 0,9739 & 0,9720 & 0,9710 & 0,9757 & 0,9614 \\
\hline$\sum$ tests & 76 & 77 & 77 & 531 & 551 & 580 & 229 \\
\hline$\sum$ tests $<1$ & 0 & 4 & 1 & 38 & 20 & 9 & 34 \\
\hline$\sum$ tests $<0,97$ & 0 & 1 & 0 & 0 & 0 & 0 & 8 \\
\hline
\end{tabular}


Table 4

Results for spatial behaviour

\begin{tabular}{|c|c|c|c|c|c|c|c|c|c|c|c|c|}
\hline & IPE 500 & & & & HEB & & & & RHS & & & \\
\hline$\overline{\lambda_{z}}$ & 0,5 & 1,0 & 1,5 & 3,0 & 0,5 & 1,0 & 1,5 & 3,0 & 0,5 & 1,0 & 1,5 & 3,0 \\
\hline$m$ & 1,0976 & 1,0901 & 1,0739 & 1,0326 & 1,0704 & 1,0704 & 1,0627 & 1,0228 & 1,0658 & 1,0844 & 1,0929 & 1,0507 \\
\hline$s$ & 0,0773 & 0,0572 & 0,0479 & 0,0365 & 0,0566 & 0,0469 & 0,0393 & 0,0329 & 0,0587 & 0,0532 & 0,0579 & 0,0450 \\
\hline max & 1,2483 & 1,2033 & 1,1726 & 1,1339 & 1,2148 & 1,1824 & 1,1451 & 1,1017 & 1,2039 & 1,2248 & 1,2475 & 1,1826 \\
\hline $\min$ & 0,9633 & 0,9718 & 0,9753 & 0,9576 & 0,9831 & 0,9648 & 0,9756 & 0,9616 & 0,9737 & 0,9854 & 0,9882 & 0,9747 \\
\hline Etests & 405 & 562 & 581 & 230 & 558 & 582 & 592 & 236 & 498 & 542 & 567 & 232 \\
\hline$\sum$ tests $<1$ & 35 & 16 & 18 & 35 & 60 & 26 & 25 & 53 & 50 & 15 & 6 & 15 \\
\hline$\sum$ tests $<0,97$ & 2 & 0 & 0 & 8 & 0 & 1 & 0 & 10 & 0 & 0 & 0 & 0 \\
\hline
\end{tabular}




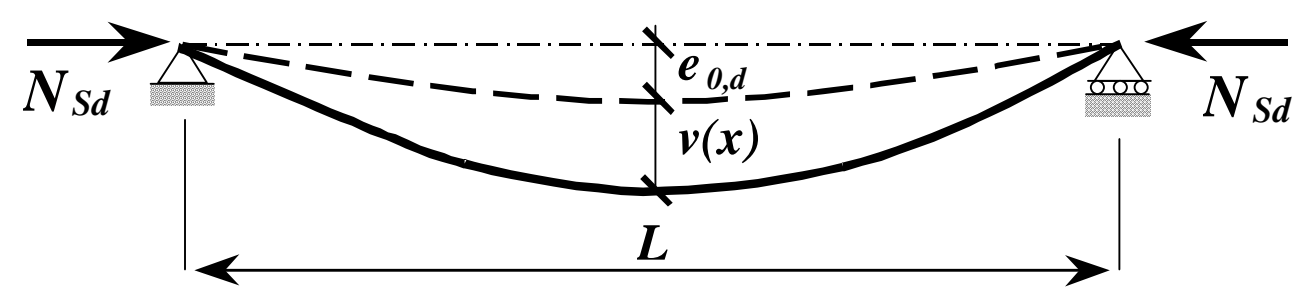

Fig. 1 Axially loaded member 


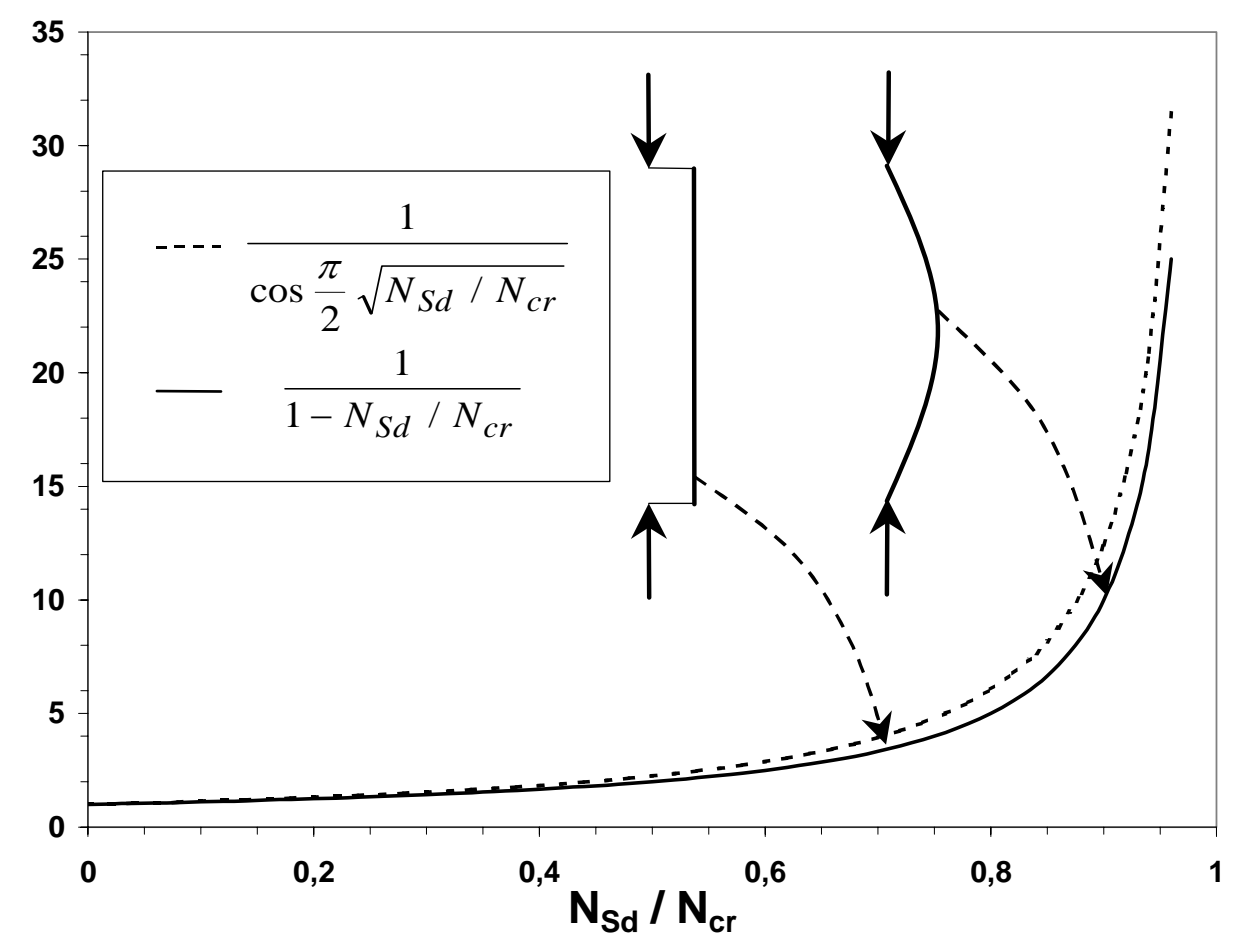

Fig. 2 Amplification factor 


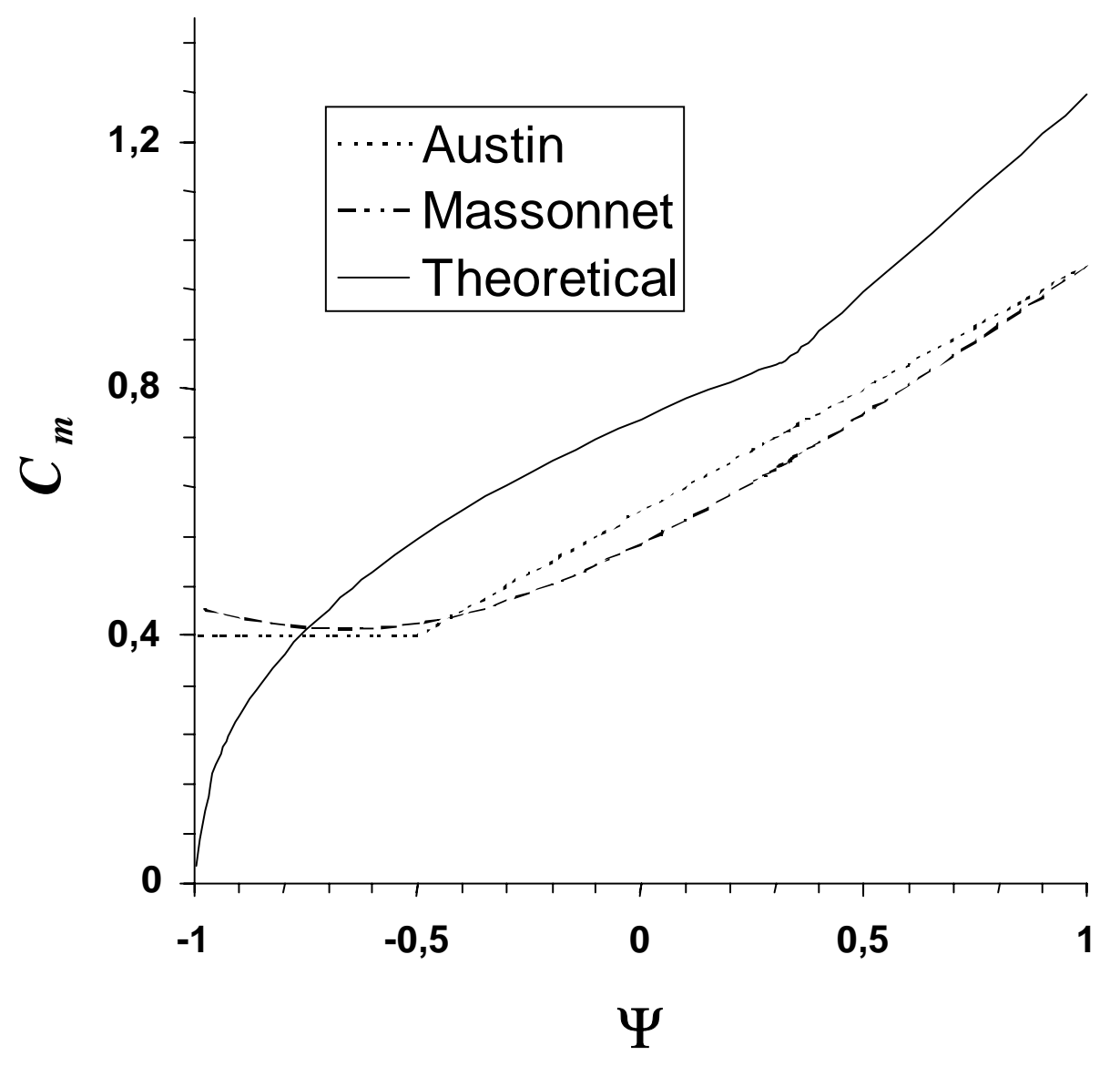

Fig. 3 Comparison of different $C_{m}$ factors 

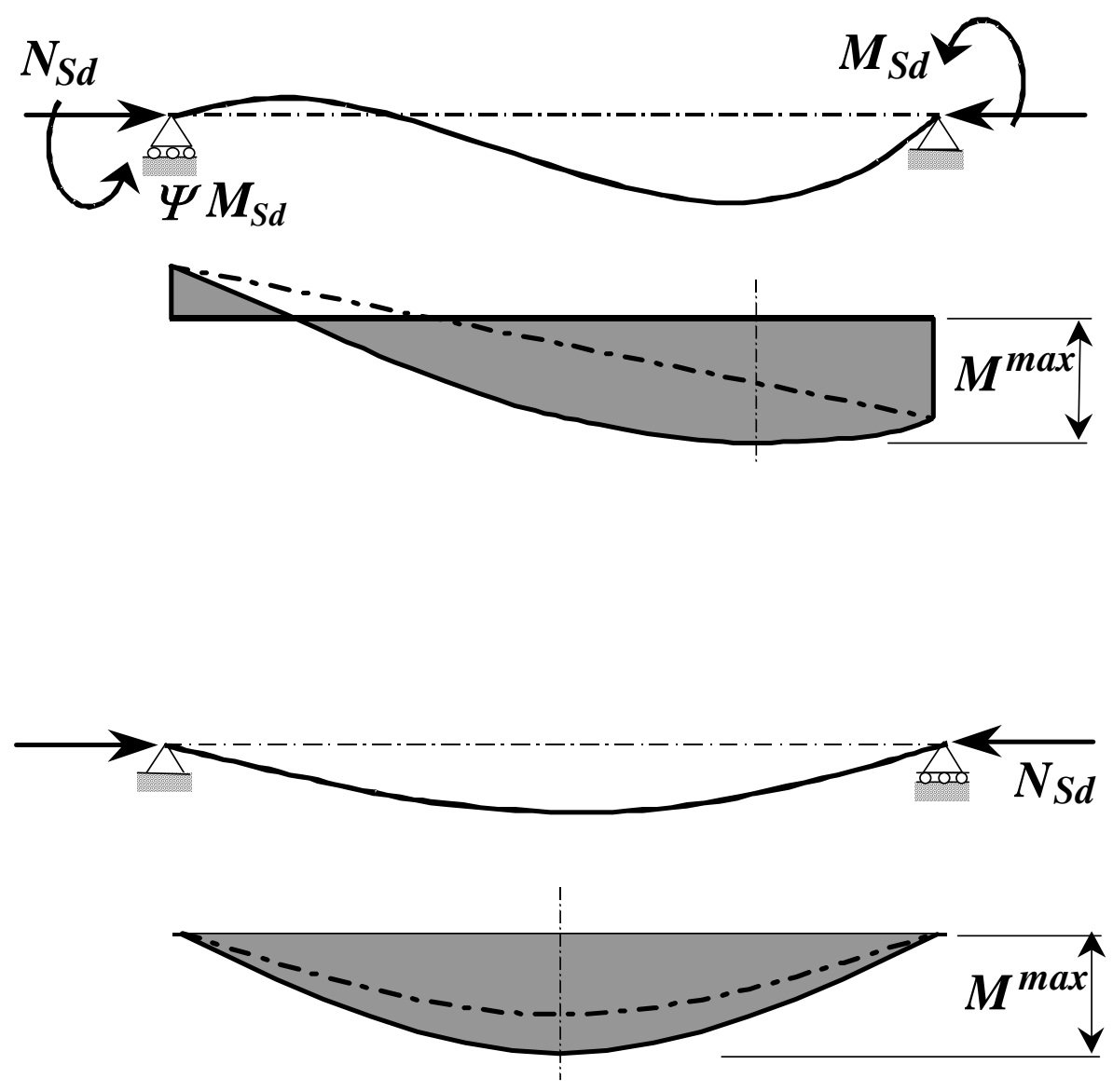

Fig. 4 Equivalent moment coefficient concept 


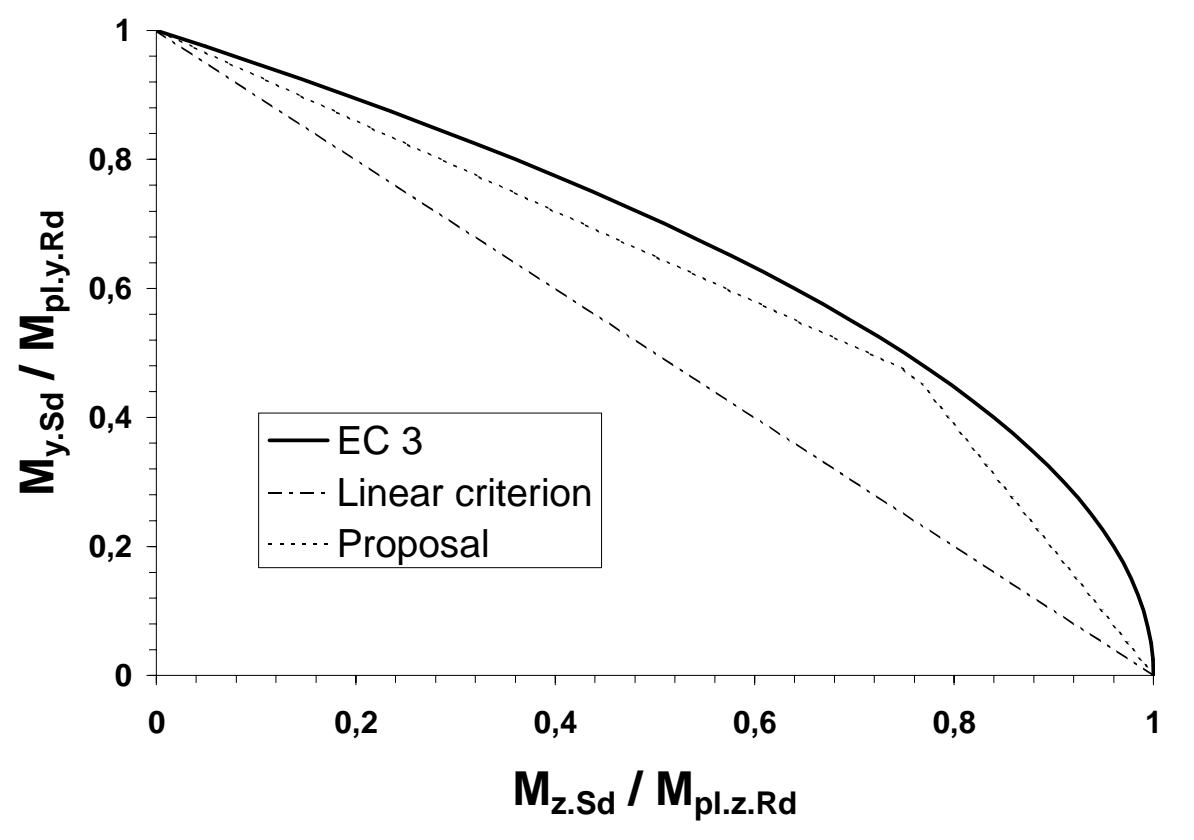

Fig. 5 Biaxial bending interaction for a classical IPE shape 


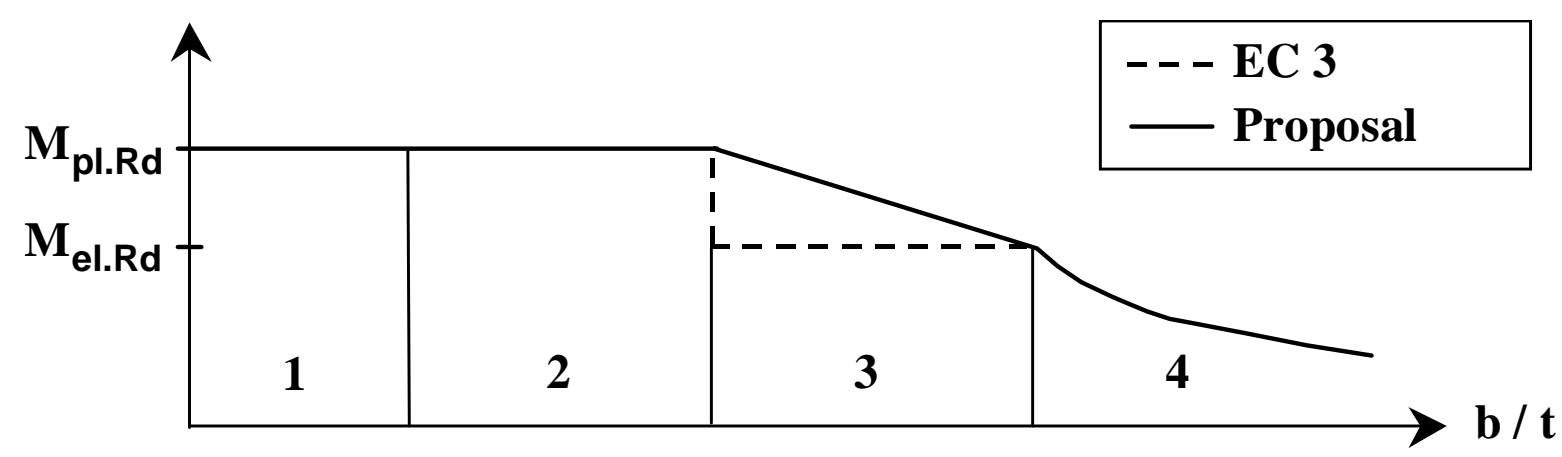

Fig. 6 Continuity between cross-section classes 


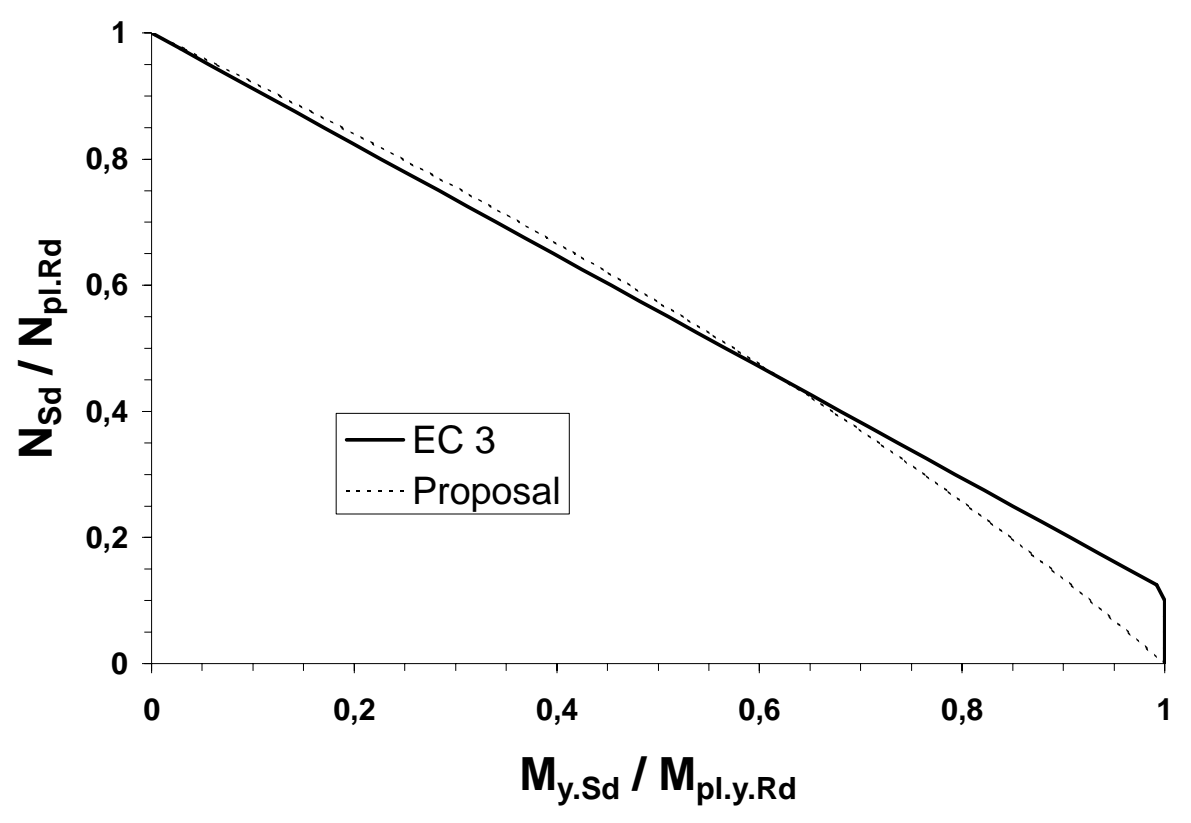

Fig. $7 \mathrm{~N}-\mathrm{M}_{\mathrm{y}}$ cross-section interaction (HEB shape) 


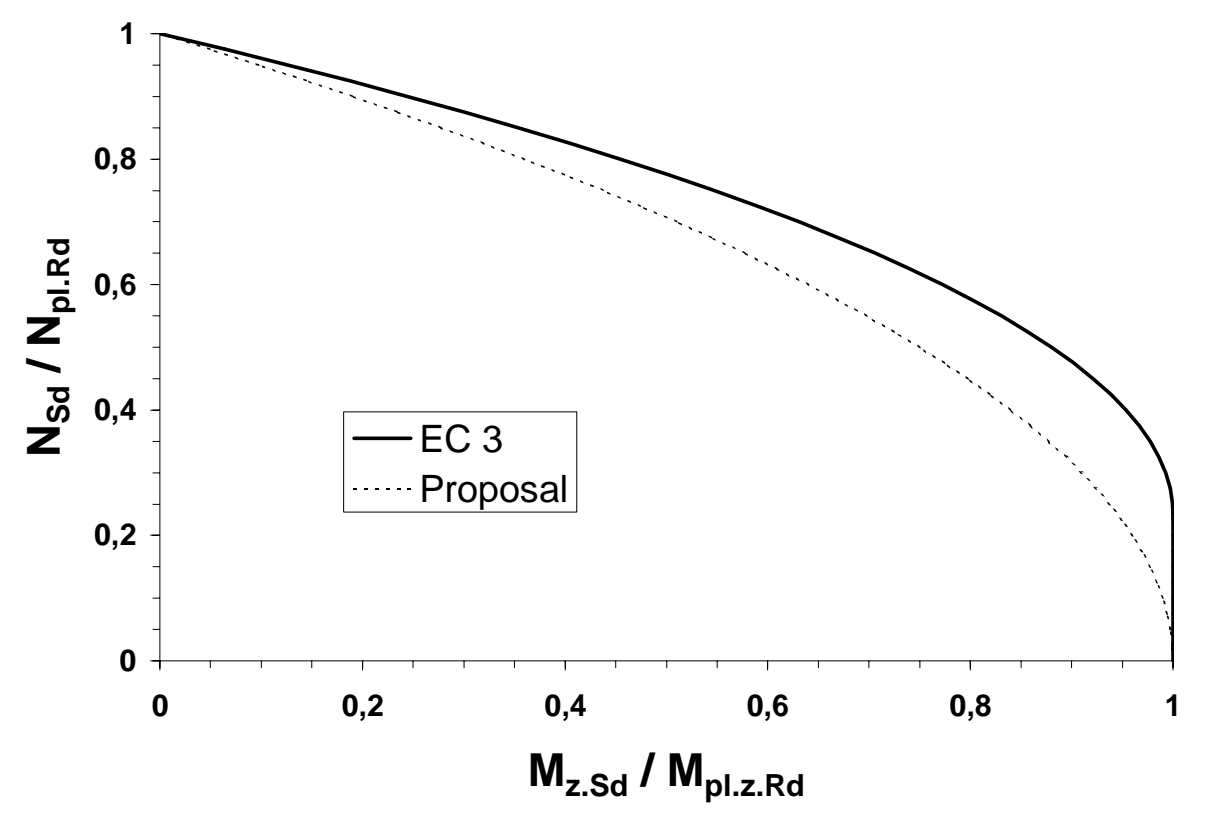

Fig. $8 \mathrm{~N}-\mathrm{M}_{\mathrm{z}}$ cross-section interaction (HEB shape) 


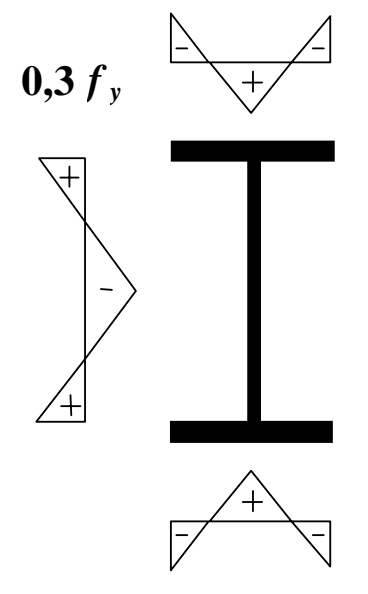

IPE - sections

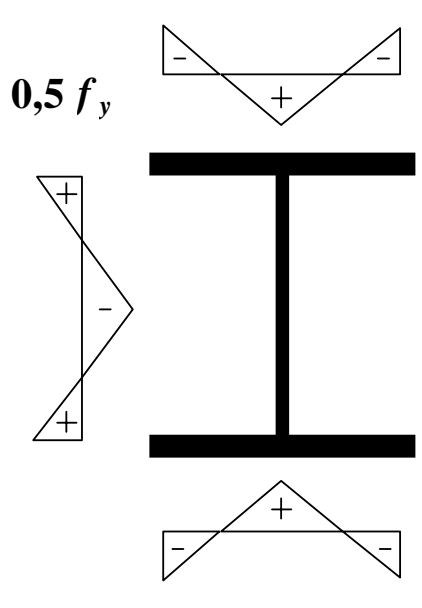

HEB - sections

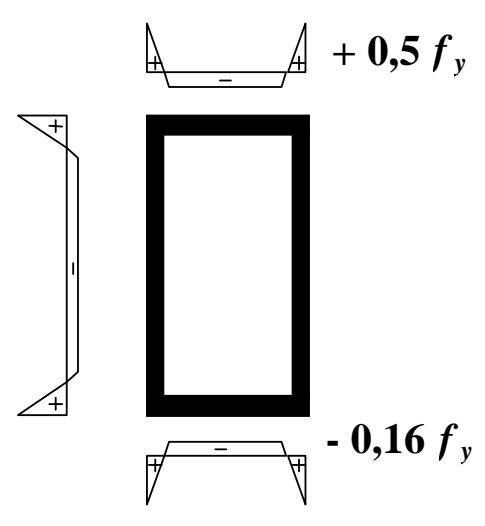

RHS - sections

Fig. 9 Residual stress diagrams used in the simulations 


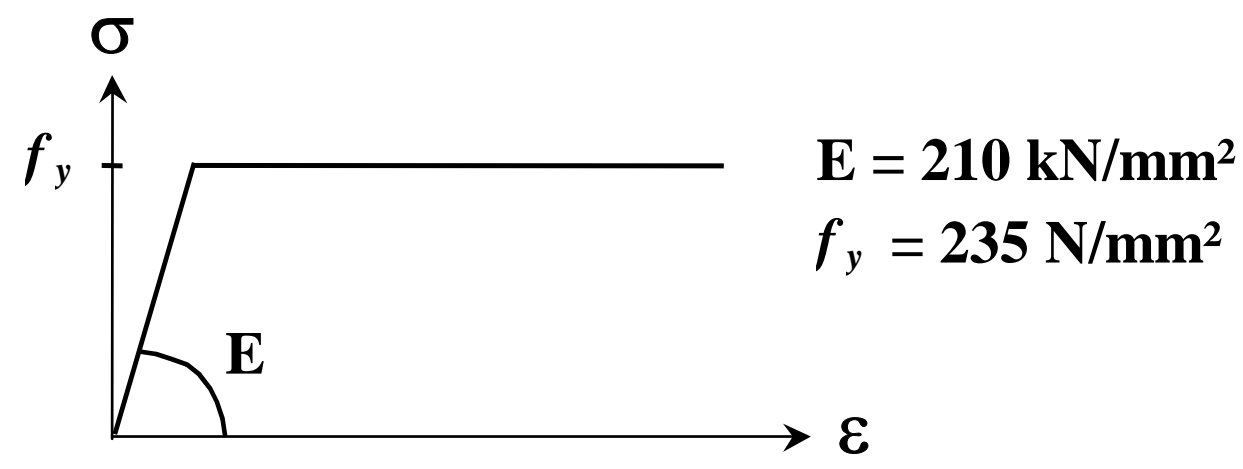

Fig. 10 Elastic-perfectly plastic constitutive law 


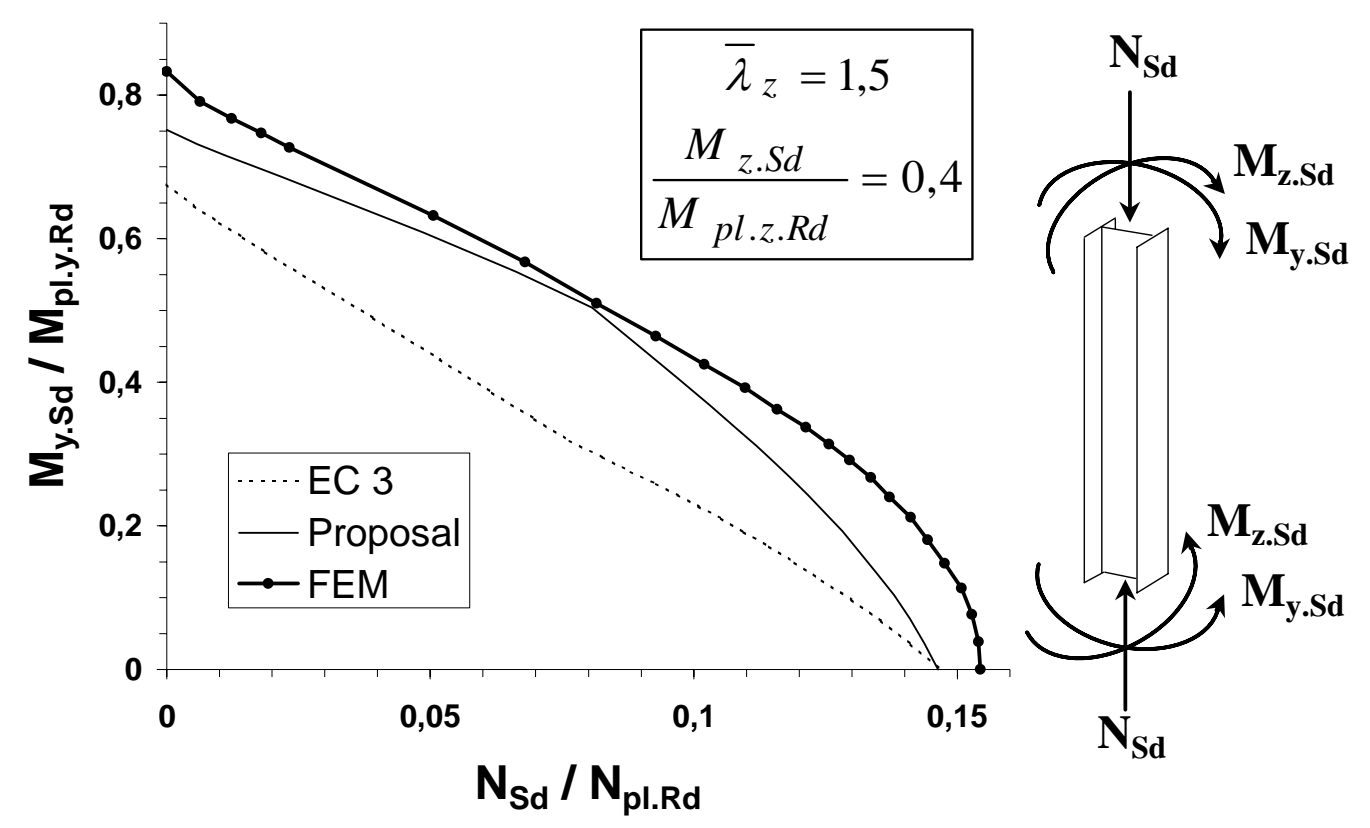

Fig. 11 Example of interaction diagram (IPE 500) 